\title{
Thrombin Injection Vs Conventional Surgical Repair in Treatment of Iatrogenic Post-Cath Femoral Artery Pseudoaneurysm (Ifap)
}

\author{
Sharkawy M*, Farghaly A and Fouad AG \\ Department of Vascular surgery, Cairo University, Egypt
}

*Corresponding author: Sharkawy M, MD Professeur of vascular surgery Kasr Ani hospital, Cairo University, Egypt, Email: sharkawy@kasralainy.edu.eg

\section{Research Article \\ Volume 4 Issue 1}

Received Date: April 18, 2020

Published Date: May 08, 2020

DOI: $10.23880 /$ ijsst-16000144

\section{Abstract}

Background: Femoral pseudo aneurysms may complicate up to $8 \%$ of vascular interventional procedures. Small pseudo aneurysms can spontaneously clot, but sometimes treatment is needed. Surgery has traditionally been considered the 'gold standard' treatment, although it is not without risk. Less invasive treatment options such as Duplex ultrasound-guided compression and percutaneous thrombin injection are available alternatives; however, their efficacy was a concern.

Aim of the study: Study the safety and efficiency of the thrombin injection in comparison to the conventional surgery as an alternative treatment of IFAPs.

Patients and Methods: 120 patients were divided into two groups A and B

Group A underwent a surgical repair.

Group B underwent US guided Thrombin injection

Results: Results were studied in terms of Success, Recurrence, Bleeding, Thrombosis, Infection, Secondary Haemorrhage and amputations

For Group A: All procedure were successful, 2 patients had wound infection, one patient had a secondary haemorrhage ended up with AKA

For Group B: 58 out of 60 procedures were successful. Only 2 needed surgical intervention.

Conclusion: Thrombin injection can be considered as a safe and considerably efficient alternative for surgery in management of femoral artery pseudo aneurysm.

Keywords: Iatrogenic complications; Femoral catheterization; Pseudoaneurysm; Surgery; Thrombin injection

\section{Introduction}

The number of percutaneous endovascular interventions performed worldwide has been growing rapidly due to important technological advances, improved long-term clinical outcomes, and, also, the lower morbidity associated with these procedures compared with traditional surgical treatment methods. The common femoral artery (CFA) is the most common access site for endovascular procedures [1].
Iatrogenic femoral artery pseudo aneurysms (IFAPs) form when an arterial puncture site fails to seal, allowing arterial blood to jet into the surrounding tissues and form a pulsatile hematoma [2]. This is a recognized and troublesome complication in patients undergoing diagnostic or therapeutic catheterization and its incidence increases when large bore sheaths and post-procedural anticoagulation therapy, and/or antiplatelet therapy are used during the intervention [3]. Complications of pseudoaneurysms include 
rupture, distal embolization, pain, femoral vein compression, neuropathy and local skin ischemia. IFAPs have historically been treated with open surgical repair, but this is not without risks especially in patients with severe cardiovascular disease [4]. Less invasive options include compression under continuous sonographic guidance with success rates ranging between $71 \%$ and $99 \%$ [4]. This technique, however, has disadvantages, including arterial occlusion, inability to treat noncompressible pseudoaneurysms, limited success in patients receiving anticoagulants, patient and operator discomfort during compression, as well as long procedure times with compression time ranging between 30 and $100 \mathrm{~min}$ [2]. The use of thrombin injection under ultrasound guidance has allowed repair of IFAPs with success rates ranging from $93 \%$ to $100 \%$ [5], and minimal complications, such as distal embolization or allergic reactions [6]. The aim of this study was to review our experience with percutaneous ultrasound-guided thrombin injection to treat IFAPs and evaluate the success of the technique in comparison to the classic conventional surgery.

\section{Patients and Methods}

In the period from March 2004 and till March 2009 we run our study on 120 patients 68 males and 52 females with age ranging from 48 to 84 all were presented with post femoral artery catheterisation pseudo aneurysm Criteria of exclusion:

- Associated lower limb ischaemia

- Infected aneurysm

- $\quad$ presence of skin necrosis

- Allergy to thrombin (For Group B)

All patients had Arterial duplex scan to confirm the diagnosis. Patients were then randomly divided into two groups:

Group A: 60 patients 33 males and 27 females underwent open surgical repair for the aneurysm after obtaining a written consent. Surgical repair was in the form of, patch plasty, interposition graft or direct repair The hospital stay of the patients underwent open surgeries varies from 3 to 21 days. All the patients had a follow up appointment with duplex scan after 4 weeks and then every 6 month for 2 years. Patients who had graft interpositions have been further followed afterwards on a year basis.

Group B: 60 patients 35 males and 25 female all underwent US guided thrombin injection under local anaesthesia as an outpatient procedure Human thrombin solution (500 U/mL) was prepared and was loaded into a $5 \mathrm{~mL}$ syringe attached to a 22-gauge spinal needle. The ultrasound transducer was placed over the pseudo aneurysm and under aseptic technique the needle was introduced with the tip in the centre of the pseudo aneurysm. Using real time sonography, thrombin was injected into the sac until thrombus filled the entire sac or the $1000 \mathrm{U}$ limit was reached. Color-Doppler was used to detect successful occlusion of the lumen. If there was flow still detected in the sac, a further $1000 \mathrm{U}$ was injected.

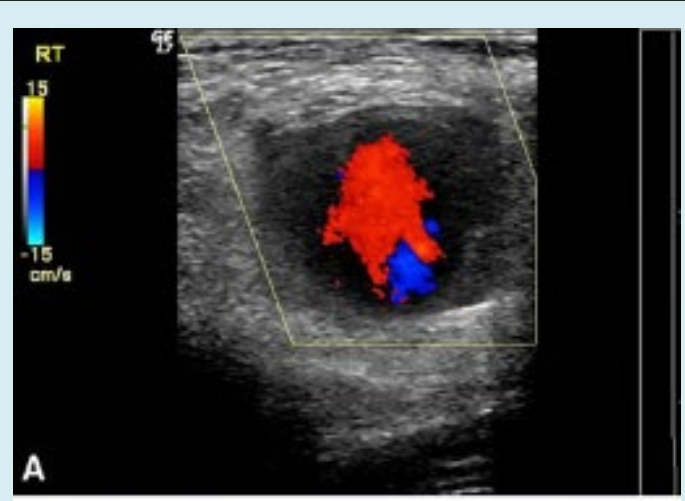

Figure 1: Duplex Scan of pseudoaneurysm showing the flow within.

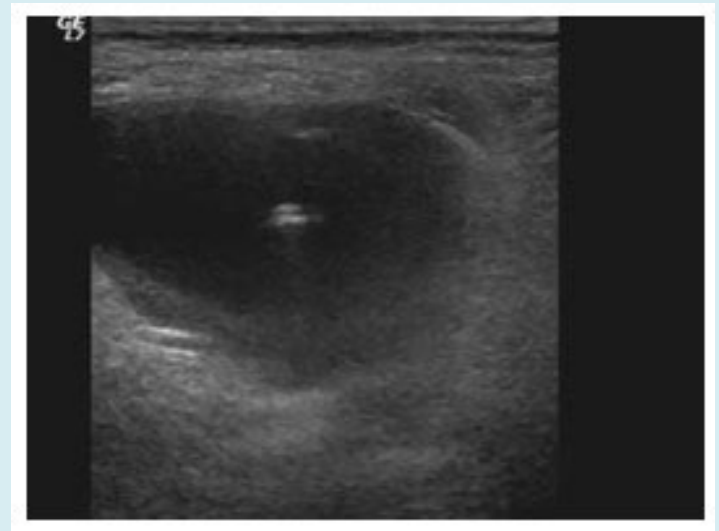

Figure 2: needle inside the aneurysm sac under US guidance

Treated patients remained in bed for $2 \mathrm{~h}$. Distal pulses were checked before and after the procedure. The patients had follow-up duplex ultrasound the day after and at four weeks following the procedure unless there was a clinical indication to perform this sooner.

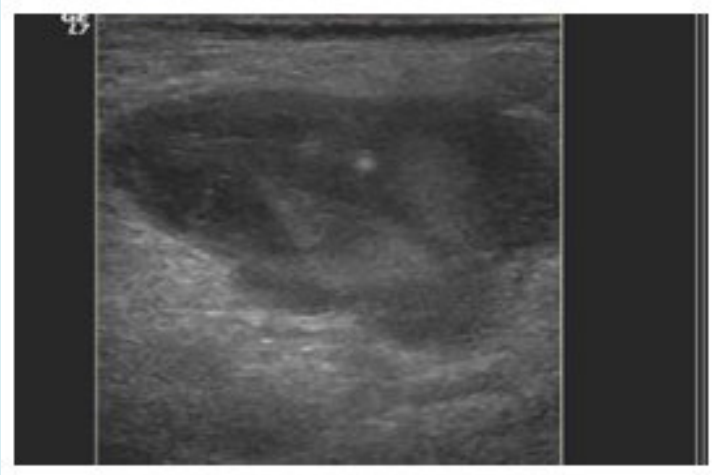

Figure 3: Thrombosis of the aneurysm after thrombin injection 


\section{International Journal of Surgery \& Surgical Techniques}

All patients had out-patient clinic follow-up at four weeks. The imaging findings and patient medical records were reviewed. The pseudo aneurysm size, site, thrombin dose injected, success and complication rates were obtained.

\section{Results}

74 patients $(62.5 \%)$ had undergone diagnostic coronary angiograms (with a 6-French sheath). 34 patients (30\%) developed IFAP following percutaneous transluminal coronary angioplasty (PTCA) with stent placement in eight (requiring a 8-French sheath), 9 (7.5\%) following lower limb angioplasty, 3 (2.5\%) after a complication from femoral catheter insertion for hemodialysis.

In 107 patients (90\%) pseudo aneurysms arose from the common femoral artery, 9 from the superficial femoralartery $(7.5 \%)$ and 3 from the profunda femoris artery(2.5\%). No associated arterio-venous fistulas were noted. The average pseudo aneurysm sac size was $3 \mathrm{~cm}$ (range $1.8-5.6 \mathrm{~cm}$ ).

\section{Group A results}

Demographic: 60 patients 33 males and 27 females with age range was between 48 and 79.48 of aneurysms occurred in the right lower limb (80\%) while $12(20 \%)$ were in the left. 52 of aneurysms raised form the CFA (86.6\%), 5 from the SFA ( $8.3 \%)$ and only 3 from the profunda (5\%).

\section{Procedures}

38 of the patients in this group had direct repair (63\%), 14 had patch plasty $(27 \%)$ while 6 had interposition graft repair $(10 \%)$.

\section{Outcome}

All procedures were considered successful in term of aneurysm repair. 4 Patients $(6.7 \%)$ had wound infections which range from mild cellulitis to superficial dehiscence 2 of the patients had post op thrombosis $3.3 \%$ one of them after patch repair and the other after an interposition graft.

Only one patient developed secondary haemorrhage (1.7\%). The same patient developed irreversible ischaemic gangrene as a result of ligation of the CFA to stop the secondary bleeding and had an above knee amputation.

\section{Group B}

A certain correlation between the diameter and the amount of the thrombin injection and a correlation between the sac diameter and the success rate were definitely noticed. All IFAPs with sac of $2.5 \mathrm{~cm}$ or less in maximum diameter required less than $500 \mathrm{U}$ (range 200-500 U) of thrombin in relation to almost $2000 \mathrm{U}$ injected in the large diameter aneurysm $(>5 \mathrm{~cm})$. Also the diameter of the aneurysm was predictive to the procedure success rate success rate as the two failed procedures were in a 5.2 and $5.4 \mathrm{~cm}$ aneurysm respectively.

\section{Demographic}

60 patients 35 males and 25 females, with age range between 51 and 84 . In 52 patients (86.7\%) the aneurysm occurred the right LL and in $8(13.3 \%)$ it was the left. 56 of aneurysms arose from the CFA (93.3\%) while 4 arose from the SFA (6.7\%).

\section{Outcome}

In 58 out of 60 patients the procedure was successful (96.7\%), 54 (90\%) needed only one session while 4 (6.7\%) needed a second session of injection, 2 patients $(3.3 \%)$ required open repair.

No patient complain of pain or required analgesia for the procedure

No post procedure infection.

No secondary haemorrhage.

No patients developed had embolic event post procedure. None of the patients had an amputation.

\section{Discussion}

Ultrasound-guided thrombin injection has become the treatment of choice for IFAPs, replacing in most centers ultrasound guided compression. Percutaneous injection of thrombin for the treatment of pseudo aneurysms was first described by Cope and Zeit in $1986 \mathrm{p}$ [6], but Walker et al. first used thrombin to treat pseudo aneurysms transluminally through a catheter [7]. The method, however, did not gain popularity until 1997 when Liau et al. successfully treated five femoral artery pseudo aneurysms with intra-vascular injection of thrombin via a catheter under continuous ultrasound guidance [8]. Ultrasound-guided thrombin injection reduces the mortality and morbidity associated with surgical repair of pseudo aneurysms. In Group A, although success rate was $100 \%$ in terms of aneurysm repair, however we recorded complications rate of $11.7 \%$ and no mortality. In their series, Lumsden, et al. demonstrated complication and mortality rates as high as $21 \%$ and $2.1 \%$, respectively, for surgical repair [9]. In our study, Group B recorded success rate in $96.7 \%$ compared to $95 \%$ in a study by Paraskevi, et al in 2009, however it is important to mention that 2 patients of the success group had further session of injection while in Paraskevi study they didn't try a second injection [10]. None of our patient developed embolic event which again agrees with the studies by Paraskevi, et al. and Kang, et al. [10] (Tables $1 \& 2$ ). 


\section{International Journal of Surgery \& Surgical Techniques}

\begin{tabular}{|c|c|c|}
\hline Demographic & Group A & Group B \\
\hline Right limb & 48 & 52 \\
\hline Left Limb & 12 & 8 \\
\hline CFA & 52 & 56 \\
\hline SFA & 5 & 4 \\
\hline
\end{tabular}

Table 1: Demographic results.

\begin{tabular}{|c|c|c|}
\hline Outcome & Group A & Group B \\
\hline Success & 60 & 58 \\
\hline Failed & 0 & 2 \\
\hline Thrombosis & 4 & 0 \\
\hline Infection & 3 & 0 \\
\hline Amputation & 1 & 0 \\
\hline Mortality & 0 & 0 \\
\hline
\end{tabular}

Table 2: Outcome results.

\section{Conclusion}

Ultrasound guided intraluminal thrombin injection is a very efficient and quite safe procedure in treating iatrogenic femoral artery pseudoaneurysm. Provided proper selection of the patients, Thrombin injection should be considered as the first choice line of treatment in iatrogenic femoral artery aneurysm.

\section{References}

1. Samal AK, White CJ (2002) Percutaneous management of access site complications. Catheter Cardiovasc Interv 57(1): 12-23.

2. Katzenschlager R, Ugurluoglu A, Ahmadi A, Hülsmann

M, Koppensteiner $\mathrm{R}$ et al. (1995)Incidence of pseudoaneurysm after diagnostic and therapeutic angiography. Radiology 195(2): 463-466.

3. Kang SS, Labropoulos N, Mansour MA, Baker WH (1998) Percutaneous ultrasound guided thrombin injection: a new method for treating postcatheterization femoral pseudoaneurysms. J Vasc Surg 27(6): 1032-1038.

4. Lenartova M, Tak T (2003) Iatrogenic pseudoaneurysm of femoral artery: case report and literature review. Clin Med Res 1(3): 243-247.

5. Ferguson JD, Whatling PJ, Martin V, Banning A (2001) Ultrasound guided percutaneous thrombin injection of iatrogenic femoral artery pseudoaneurysms after coronary angiography and intervention. Heart 85(4): E5.

6. Morgan R, Belli AM (2003) Current treatment methods for postcatheterization pseudoaneurysms. J Vasc Interv Radiol 14(6): 697-710.

7. Cope C, Zeit R (1986) Coagulation of aneurysms by direct percutaneous thrombin injection. Am J Roentgenol 147(2): 383-387.

8. Walker TG, Geller SC, Brewster DC (1987) Transcatheter occlusion of a profunda femoral artery pseudoaneurysm using thrombin. Am J Roentgenol 149(1): 185-186.

9. Liau CS, Ho FM, Chen MF (1997) Treatment of iatrogenic femoral artery pseudoaneurysms with percutaneous thrombin injection. J Vasc Surg 26(1): 18-23.

10. Vlachou PA, Karkos CD, Bains S, McCarthy MJ, Fishwick G, et al. (2011) Percutaneous ultrasound-guided thrombin injection for the treatment of iatrogenic femoral artery pseudoaneurysms. Eur J Radiol 77(1): 172-174. 